\title{
The evaluation of nailfold capillaroscopy pattern in patients with fibromyalgia
}

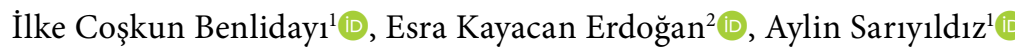 \\ ${ }^{1}$ Department of Physical Medicine and Rehabilitation, Çukurova University Faculty of Medicine, Adana, Turkey \\ ${ }^{2}$ Department of Internal Medicine, Division of Rheumatology, Çukurova University Faculty of Medicine, Adana, Turkey
}

\begin{abstract}
Objectives: This study aims to evaluate nailfold capillaroscopic pattern in patents with fibromyalgia and to assess the relation of capillaroscopic parameters with clinical variables and disease-related measures.

Patients and methods: This cross-sectional, case-control study included 60 participants (4 males, 56 females; mean age: $44.0 \pm 8.2$ years; range, 26 to 64 years) between August 2019 and November 2019. All participants were divided into two groups as the primary fibromyalgia group $(n=30)$ who met the 2016 modified American College of Rheumatology Diagnostic Criteria for Fibromyalgia and the control group ( $n=30)$ consisting of age- and sex-matched healthy individuals. Nailfold capillaroscopy was performed by a digital microscope under a magnification of 200x. Capillary density, capillary loop diameter, number of dilated, giant and neoangiogenic capillaries, capillary shape, number of avascular areas, micro-aneurysms and micro-hemorrhages were evaluated by an assessor who was blind to the group allocation. In the fibromyalgia group, Widespread Pain Index, Symptom Severity Scale scores, and Fibromyalgia Severity scores were calculated. Health status and presence of benign joint hypermobility syndrome (BJHS) were evaluated using the Fibromyalgia Impact Questionnaire (FIQ) and revised Brighton criteria, respectively. Results: Of the capillaroscopic parameters, the mean capillary loop diameter, number of micro-aneurysms, avascular areas, and neoangiogenic capillaries were significantly higher in the patient group compared to the controls ( $p<0.001, p=0.016, p=0.038$, and $p=0.04$, respectively). Nailfold capillaroscopic findings did not significantly differ between the patients with $(n=16)$ and without concomitant BJHS ( $n=14)$. Of the disease-related measures, only FIQ score showed a weak correlation with the number of dilated capillaries $(p=0.324)$.
\end{abstract}

Conclusion: Patients with fibromyalgia have distinct capillaroscopic patterns than healthy population. Capillaroscopic features, in general, are not related to clinical variables and disease-related measures.

Keywords: Capillaries, capillaroscopy, fibromyalgia, nailfold capillaroscopy.

Fibromyalgia is a chronic rheumatic condition affecting a large proportion of individuals worldwide. A survey conducted in five European countries demonstrated the estimated point prevalence as ranging from $2.9 \%$ to $4.7 \%$, depending on the screening questionnaire used. ${ }^{1}$ However, much higher rates were reported in patients with inflammatory rheumatic conditions, such as rheumatoid arthritis and spondyloarthritis. ${ }^{2-4}$ Despite its high recognition among physicians, there are still unsolved points in terms of the etiology and pathogenesis of the disease. For a better understanding of the disease etiopathogenesis, clinical picture of the disease and each individual symptom/sign should be examined thoroughly.

Fibromyalgia has a wide range of clinical symptoms including chronic widespread pain,

Received: August 04, 2020 Accepted: December 01, 2020 Published online: January 15, 2021

Correspondence: Illke Coşkun Benlidayı, MD. Çukurova Üniversitesi Tıp Fakültesi Fiziksel Tıp ve Rehabilitasyon Anabilim Dalı, 01790 Sarıçam, Adana, Türkiye. Tel: +90 538 - 5453937 e-mail: icbenlidayi@hotmail.com

Citation:

Coşkun Benlidayı I, Kayacan Erdoğan E, Sarıyıldız A. The evaluation of nailfold capillaroscopy pattern in patients with fibromyalgia. Arch Rheumatol 2021;36(3):341-348. 
fatigue, unrefreshed waking, cognitive problems, headache, abdominal pain/cramps, and mood disorders. ${ }^{5}$ Vasospastic symptoms are also common in patients with fibromyalgia. ${ }^{6}$ Besides, pain -the cardinal symptom of fibromyalgiamay be related to muscle hypoperfusion induced by regional vasomotor dysregulation. ${ }^{7}$ In this context, a number of studies have focused on the microcirculation abnormalities in patients with fibromyalgia, showing diminished microcirculation in fibromyalgia. ${ }^{6,8-10}$ However, there is a limited number of studies using distinct methodologies. Besides, evidence regarding the potential relation between disease severity and microcirculatory abnormalities remains limited.

Given this gap in the literature, the primary objective of the current study was to evaluate nailfold capillary morphology in patients with fibromyalgia. Secondary objectives were (i) to examine the relationship between capillaroscopic findings and disease-related variables (i.e., pain intensity, disease severity and current health status) and (ii) to identify the potential impact of concomitant joint hypermobility on capillaroscopic abnormalities.

\section{PATIENTS AND METHODS}

This cross-sectional, case-control study was conducted at Çukurova University Faculty of Medicine between August 2019 and November 2019. A total of 60 participants (4 males, 56 females; mean age: $44.0 \pm 8.2$ years; range, 26 to 64 years) were included in the study. All participants were divided into two groups as the primary fibromyalgia group ( $\mathrm{n}=30$ ) who met the 2016 modified American College of Rheumatology (ACR) Diagnostic Criteria for Fibromyalgia ${ }^{5}$ and the control group $(n=30)$ consisting of age- and sex-matched healthy individuals. Exclusion criteria were as follows: having inflammatory rheumatic diseases, concomitant diseases with a potential risk for microvascular involvement, any known vasculopathy, and the use of vasodilatory medications. A written informed consent was obtained from each participant. The study protocol was approved by the Ethics Committee of Çukurova University Faculty of Medicine (approval date: 05/07/2019, no: 90/13).
The study was conducted in accordance with the principles of the Declaration of Helsinki.

Demographic variables including age and sex of each participant were recorded. In the patient group, disease duration (months), Widespread Pain Index (WPI), Symptom Severity Scale (SSS) scores, and Fibromyalgia Severity (FS) scores were also noted. The WPI and SSS scores were evaluated as described in the 2016 modified ACR diagnostic criteria. The FS scores were calculated as the sum of WPI and SSS scores. ${ }^{5}$

The patients' current health status was evaluated using the Fibromyalgia Impact Questionnaire (FIQ). ${ }^{11,12}$ This self-administered tool consists of 10 items. The first item contains 11 questions measuring physical functioning on a 4-point Likert scale (0-3). The following two items ask the number of days (0-7) that the patient felt well (item 2) and was unable to work (item 3) due to fibromyalgia. The remaining seven items (item 4-10) rate work difficulty, pain, fatigue, morning tiredness, stiffness, anxiety, and depression (0-10). The total FIQ score ranges between 0 and 100. Higher scores represent poor current health status. The patients were also evaluated in terms of joint hypermobility using the Beighton scoring system. ${ }^{13}$ Diagnosis of benign joint hypermobility syndrome (BJHS) was made based on the revised Brighton criteria. ${ }^{14}$

Nailfold capillaroscopy was performed using a digital microscope (Dino-Lite CapillaryScope 200, Naarden, Netherlands) and related software program (The DinoCapture v2.0 software from AnMo Electronics Corp., Taiwan). The methodology was adopted and modified from the previously published studies by Hosking et al. ${ }^{15}$ and Sulli et al. ${ }^{16}$ The procedure was performed by a certified physician who was blinded to the study groups. Prior to the procedure, the participants rested for 15 to 20 min in a temperature-stable outpatient clinic room. Immersion oil was applied to the nailfold epidermis of the second-fifth digits of both hands. Each nailfold was evaluated under a magnification of $200 \times$, divided into four consecutive 1-mm fields and related images were recorded (Figure 1). Following parameters were evaluated on each field: (i) capillary density/number, (ii) capillary loop diameter, (iii) dilated capillaries, 


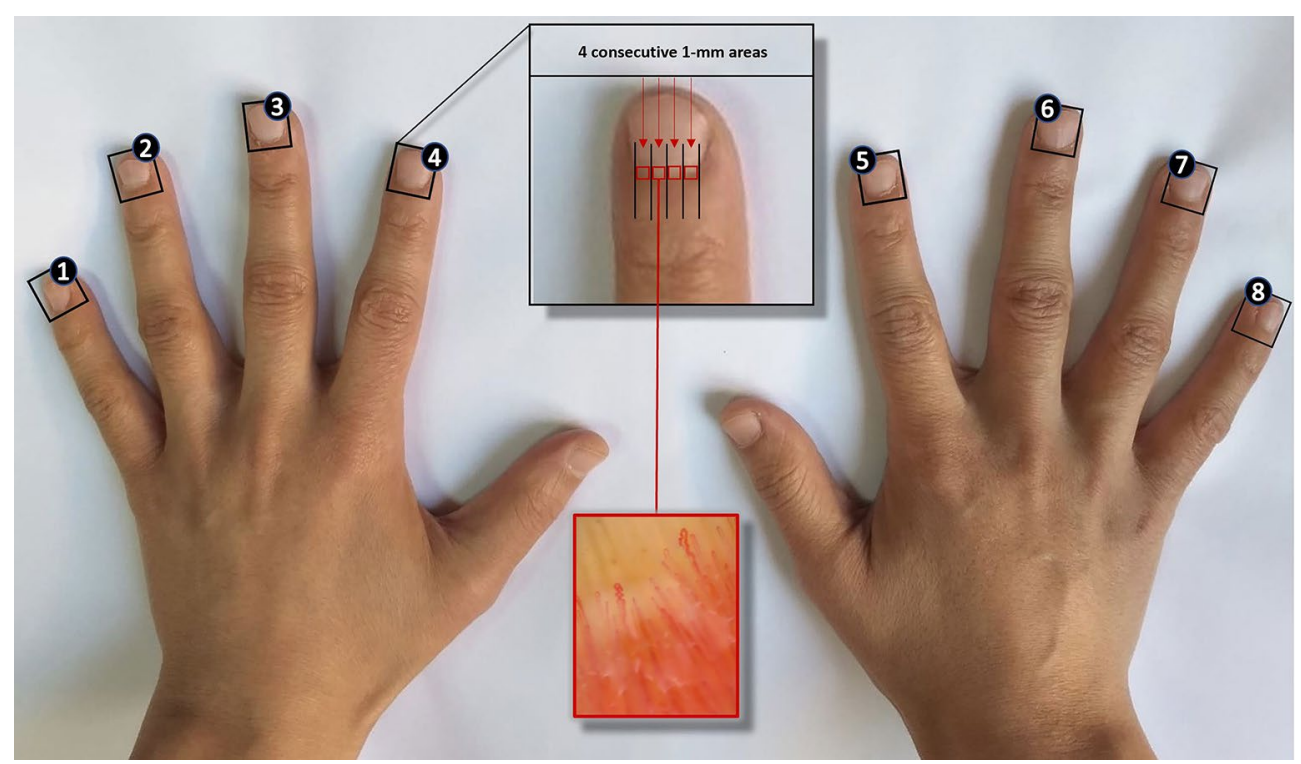

Figure 1. Methodology of nailfold capillaroscopy.

(iv) giant capillaries, (v) neoangiogenic capillaries, (vi) micro-hemorrhages, (vii) avascular areas, (viii) micro-aneurysms, and (ix) the dominant capillary shape. The number or presence of each capillaroscopic parameter was recorded on a standardized form. The total value of each parameter was calculated from the results of four consecutive fields on each digit. As for the capillary density and capillary loop diameter, a mean score value was obtained from all eight digits. As for the remaining capillaroscopic parameters including dilated capillaries, giant capillaries, neoangiogenic capillaries, micro-hemorrhages, avascular areas, and micro-aneurysms, the total number of each variable was calculated from eight digits. Capillaries with a diameter greater than $>20 \mu \mathrm{m}$ and $>50 \mu \mathrm{m}$ were defined as dilated and giant capillary, respectively. Distinct areas where two or more capillaries were missing in the nailfold were defined as avascular areas. An irregularly enlarged, circumscribed increase of the capillary loop diameter was considered micro-aneurysms. Dominant capillary shape was classified into three groups as normal, crossed, and tortuous. ${ }^{15,17}$

\section{Statistical analysis}

Power analysis and sample size calculation were performed using the G*Power version 3.1.0 software (Heinrich-Heine-Universität Düsseldorf,
Düsseldorf, Germany). The effect size was based on the minimal statistically important difference in capillary diameter between patients and controls. ${ }^{9}$ The alpha level and power were set as 5\%(0.05) and 80\%, respectively. Statistical analysis was performed using the IBM SPSS version. 20.0 software (IBM Corp, Armonk, NY, USA). The distribution of data was tested using the Shapiro-Wilk test and related histograms. Descriptive data were expressed in mean and standard deviation (SD), median (min-max), or number and frequency. Comparative analysis of normally-distributed continuous variables between fibromyalgia and control groups was performed by the independent samples t-test. Inter-group comparison of continuous variables with skewed distribution was done using the Mann-Whitney U test. The Fisher's exact test was used to compare categorical variables between the patient and control groups. The correlation of capillaroscopic parameters with clinical variables was analyzed using the Spearman's correlation analysis. A $p$ value of $<0.05$ was considered statistically significant.

\section{RESULTS}

Demographic, clinical, and capillaroscopic characteristics of the study groups are shown in Table 1. Accordingly, the patients and control 
Table 1. Demographic, clinical, and capillaroscopic characteristics of study groups $(n=60)$

\begin{tabular}{|c|c|c|c|c|c|c|c|c|c|c|c|}
\hline & & & ontrol group & $(n=30)$ & & & Fibr & omyalgia grou & p $(n=30)$ & & \\
\hline & $\mathrm{n}$ & $\%$ & Mean \pm SD & Median & Min-Max & $\mathrm{n}$ & $\%$ & Mean \pm SD & Median & Min-Max & $p$ \\
\hline Age (year) & & & $43.5 \pm 9.0$ & & & & & $44.5 \pm 7.5$ & & & 0.631 \\
\hline $\begin{array}{l}\text { Sex } \\
\text { Female } \\
\text { Male }\end{array}$ & $\begin{array}{c}29 \\
1\end{array}$ & $\begin{array}{c}96.7 \\
3.3\end{array}$ & & & & $\begin{array}{c}27 \\
3\end{array}$ & $\begin{array}{l}90 \\
10\end{array}$ & & & & 0.305 \\
\hline $\begin{array}{l}\text { FM-related parameters } \\
\text { Disease duration (month) } \\
\text { WPI } \\
\text { SSS score } \\
\text { FS score } \\
\text { FIQ score }\end{array}$ & & & $\begin{array}{l}- \\
- \\
-\end{array}$ & - & & & & $\begin{array}{c}12.7 \pm 3.3 \\
8.7 \pm 2.2 \\
21.3 \pm 4.6 \\
62.9 \pm 15.1\end{array}$ & 36 & $5-120$ & $\begin{array}{l}- \\
- \\
- \\
-\end{array}$ \\
\hline $\begin{array}{l}\text { Capillaroscopic parameters } \\
\text { Capillary density } \\
\text { Capillary loop diameter } \\
\text { Dilated capillaries } \\
\text { Giant capillaries } \\
\text { Neoangiogenic capillaries } \\
\text { Micro-hemorrhages } \\
\text { Avascular areas } \\
\text { Micro-aneurysms }\end{array}$ & & & $\begin{array}{l}9.3 \pm 0.9 \\
8.5 \pm 1.0\end{array}$ & $\begin{array}{l}0 \\
0 \\
0 \\
0 \\
0 \\
0\end{array}$ & $\begin{array}{l}0-3 \\
0-0 \\
0-0 \\
0-0 \\
0-0 \\
0-1\end{array}$ & & & $\begin{array}{c}9.0 \pm 1.1 \\
10.1 \pm 1.0\end{array}$ & $\begin{array}{l}0 \\
0 \\
0 \\
0 \\
0 \\
0\end{array}$ & $\begin{array}{l}0-6 \\
0-1 \\
0-4 \\
0-3 \\
0-3 \\
0-9\end{array}$ & $\begin{array}{c}0.375 \\
<0.001 \\
0.138 \\
0.317 \\
0.040 \\
0.078 \\
0.038 \\
0.016\end{array}$ \\
\hline $\begin{array}{l}\text { Dominant capillary shape } \\
\text { Normal } \\
\text { Crossed } \\
\text { Tortuous }\end{array}$ & $\begin{array}{c}26 \\
4 \\
0\end{array}$ & $\begin{array}{c}86.7 \\
13.3 \\
0\end{array}$ & & & & $\begin{array}{c}26 \\
4 \\
0\end{array}$ & $\begin{array}{c}86.7 \\
13.3 \\
0\end{array}$ & & & & $>0.999$ \\
\hline
\end{tabular}

SD: Standard deviation; Min: Minimum; Max: Maximum; FM: Fibromyalgia; WPI: Widespread Pain Index; SSS: Symptom Severity Scale; FS: Fibromyalgia Severity; FIQ: Fibromyalgia Impact Questionnaire.

groups were similar in terms of age and sex ( $p=0.631$ and $p=0.305$, respectively). In the patient group, median disease duration was 36 (range, 5 to 120) months. The mean WPI, SSS, $\mathrm{FS}$, and FIQ scores were $12.7 \pm 3.3,8.7 \pm 2.2$, $21.3 \pm 4.6$, and $62.9 \pm 15.1$, respectively.
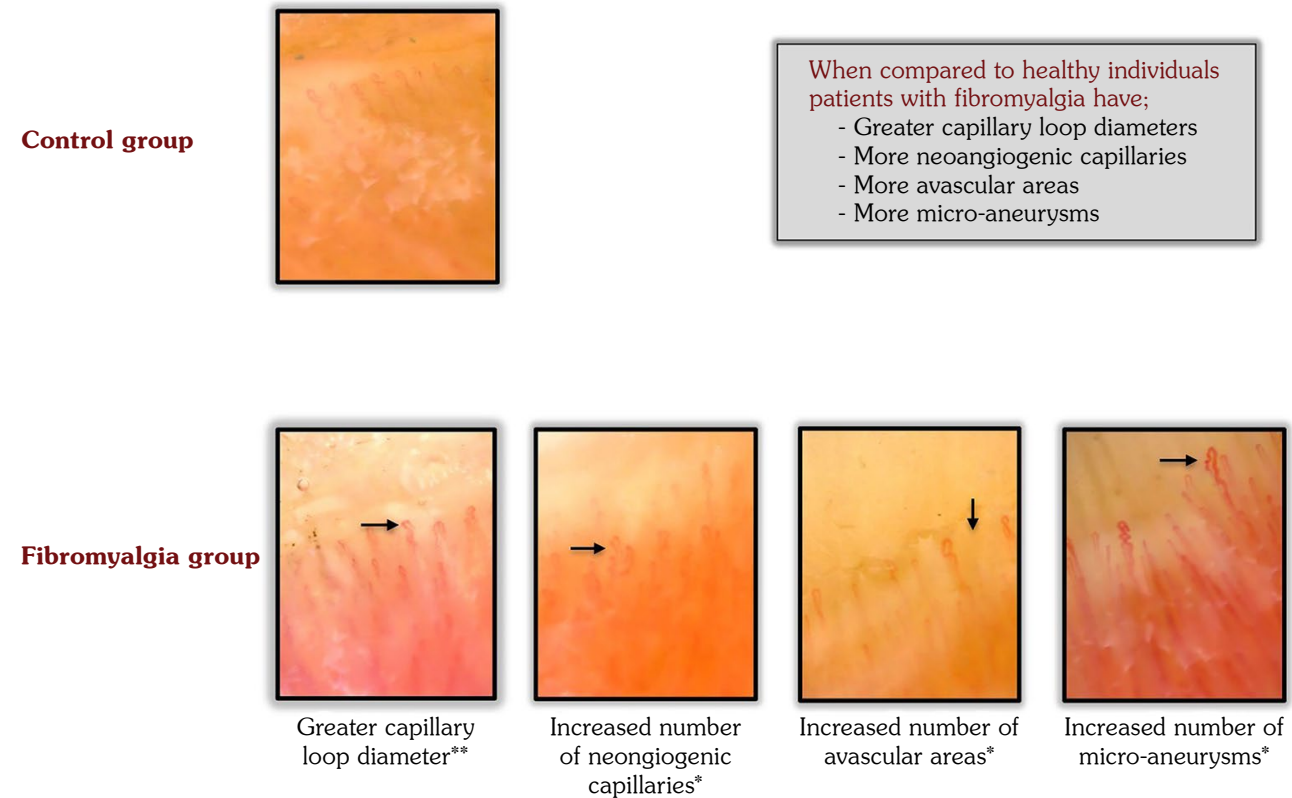

Figure 2. Comparison of capillaroscopic findings between patient and control groups.

${ }^{*} \mathrm{p}<0.05 ;{ }^{* *} \mathrm{p}<0.001$ 
Table 2. Correlation of capillaroscopic findings with disease-related parameters in patients with fibromyalgia $(n=30)$

\begin{tabular}{lccccc}
\hline Variables & Disease duration & WPI & SSS score & FS score & FIQ score \\
\hline Capillary density & -0.121 & 0.219 & -0.128 & 0.072 & -0.195 \\
Capillary loop diameter & 0.174 & -0.005 & -0.035 & -0.012 & 0.202 \\
Dilated capillaries & 0.146 & 0.156 & 0.038 & 0.142 & $0.371^{*}$ \\
Giant capillaries & 0.281 & 0.141 & -0.195 & 0.000 & 0.161 \\
Neoangiogenic capillaries & 0.057 & -0.100 & -0.004 & -0.062 & 0.068 \\
Micro-hemorrhages & 0.223 & 0.058 & -0.156 & -0.025 & 0.152 \\
Avascular areas & -0.115 & -0.107 & -0.049 & -0.101 & 0.007 \\
Micro-aneurysms & -0.022 & -0.261 & -0.327 & -0.279 & -0.091 \\
\hline Values represent Spearman's rho; WPI: Widespread Pain Index; SSS: Symptom Severity Scale; FS: Fibromyalgia Severity; FIQ: \\
Fibromyalgia Impact Questionnaire; ${ }^{*}$ p $<0.05$.
\end{tabular}

The comparison of capillaroscopic parameters between the patients and controls is given in Table 1 and Figure 2. Accordingly, the mean capillary diameter was significantly greater in fibromyalgia patients than the controls $(10.1 \pm 1.0$ us.vs. $8.5 \pm 1.0$, respectively; $\mathrm{p}<0.001)$. However, the mean capillary density showed no statistically significant difference between the two groups $(p=0.375)$. Numbers of micro-aneurysms, avascular areas, and neoangiogenic capillaries were significantly higher in the fibromyalgia group than the control group $(p=0.016, p=0.038$, and $p=0.04$, respectively). On the other hand, the number/presence of dilated capillaries, giant capillaries, and micro-hemorrhages did not significantly differ between the patients and controls $(p=0.138, p=0.317$, and $p=0.078$, respectively). In terms of dominant capillary shape, there was no significant difference between the groups. The results of the subgroup analysis in patients with fibromyalgia revealed that fibromyalgia patients with concomitant BJHS $(n=16)$ and those without BJHS $(n=14)$ had similar capillaroscopic findings.

The results of the correlation analysis of capillaroscopic findings with disease-related parameters are presented in Table 2 . Accordingly, the number of dilated capillaries showed a weak correlation with the FIQ score $\left(r_{s}=0.371, \quad p=0.044\right)$. Other capillaroscopic measures were not correlated with disease-related variables.

\section{DISCUSSION}

The present study revealed that patients with fibromyalgia had morphologic abnormalities in microcirculatory system, including the increased capillary diameter and increased number of micro-aneurysms, avascular areas, and neoangiogenic capillaries. Such microcirculatory morphology is common in autoimmune and/or inflammatory rheumatic conditions. Nevertheless, it would be regarded as an unexpected finding in fibromyalgia and would lead to several questions in terms of disease etiology and pathophysiology: Does fibromyalgia has autoimmune/inflammatory background? Are these microcirculatory abnormalities related to symptoms and/or symptom severity in fibromyalgia? Could they be potential targets for treatment?

The endothelium, the essential organ for the regulation of vascular morphology, vascular tone and trafficking of numerous blood-borne molecules, is composed of a single layer of endothelial cells. The endothelium responds to various signals including mechanical, thermal, and chemical stimuli. A direct/indirect increase in any of these stimuli facilitates endothelial cell activation which, in turn, leads to endothelial dysfunction and the phenotypical modulation of the microvascular structure. Chemical stimuli including local factors such as pro-inflammatory mediators are one of the main confounders of endothelial dysfunction. ${ }^{18}$ Microvascular changes can occur as 
a result of inflammatory/immune damage, which is triggered by the recruitment of inflammatory mediators and immune cells in the endothelial tissue. Cytotoxic T cells and anti-endothelial cell, anti-angiotensin II and anti-endothelin type-A receptor antibodies are the main factors involved in endothelial cell apoptosis and/or secretion of chemotactic mediators, such as monocyte chemoattractant protein-1, interleukin (IL)-1, IL-6 and IL-8. ${ }^{19}$ Thus, the combination of immune and inflammatory dysregulation leads to endothelial dysfunction, apoptosis, and loss of capillaries. The findings of the current study may partly support the suggestion about the role of inflammatory/autoimmune pathways in fibromyalgia, as microvascular abnormalities show similarity with those observed in inflammatory/autoimmune rheumatic diseases. During the last couple of decades, researchers have focused on the potential role of inflammation in fibromyalgia etiopathogenesis. ${ }^{20-23}$ Although inflammation is not directly related to fibromyalgia, the results of these studies do not ignore its potential effect in disease background. ${ }^{24}$ Several cytokines such as IL- 6 and IL- 8 showed increased levels in patients with fibromyalgia. ${ }^{21,25-27}$ The increased serum/cerebrospinal levels of these mediators have been linked to peripheral/central neuroinflammation in fibromyalgia. Such an increase may be also responsible for structural disorganization in microvascular tissue, which is preceded by endothelial activation and dysregulation. ${ }^{18}$ In this context, the potential relationship between pro-inflammatory mediators and nailfold capillary abnormalities still remains to be studied thoroughly.

Nailfold capillaroscopic changes in fibromyalgia syndrome may be also explained through sympathetic dysregulation. Abnormal adrenergic receptor reactivity and inadequate release of vasoactive mediators/neuropeptides are potential drivers of microcirculatory abnormalities. ${ }^{19}$ The role of adrenergic receptors in fibromyalgia pathogenesis has been widely studied. Adrenergic receptor polymorphisms, which may affect responses to adrenergic glands, have been demonstrated in patients with fibromyalgia. ${ }^{28}$ On the other hand, neuropeptide levels (neuropeptide Y, substance $\mathrm{P}$ and corticotropin-releasing hormone) are also increased in fibromyalgia. ${ }^{20,29}$ Overall, recurrent vasospasm may induce endothelial dysfunction and such microstructural changes observed in our study sample.

One potential explanation of microvasculopathy in fibromyalgia may be the increased peripheral accumulation of reactive oxygen radicals. Serum levels of oxidative stress markers are elevated, mitochondrial deoxyribonucleic acid content and coenzyme Q10 are decreased in patients with fibromyalgia. ${ }^{30,31}$ These factors along with the reduced levels of anti-oxidant enzymes lead to an increase in free radicals and oxidative stress in fibromyalgia. ${ }^{30}$ Besides, there is an interplay among oxidative stress, hypoxia, and inflammation. ${ }^{32,33}$ Endothelial cell injury, induced by oxidative stress and inflammation, may explain some capillary abnormalities including increased number of avascular areas. On the other hand, capillary neoangiogenesis may occur as a result of hypoxia-induced neovascularization.

As a secondary objective, the present study evaluated the clinical relevance of these capillaroscopic findings. Capillaroscopic variables did not significantly differ between the patients with and without concomitant BJHS. Besides, fibromyalgia-related variables were not associated with capillaroscopic parameters, except for a weak linear correlation between health status and number of dilated capillaries. However, the mean FIQ score of the study population was 62.9, and 83.3\% had FIQ score above the average cut off ( $>50)$. Due to this homogeneity, it is difficult to draw a firm conclusion about the correlation of health status with capillaroscopic findings.

In daily clinical practice, nailfold capillaroscopy is often used for the differentiation of primary Raynaud's phenomena from secondary Raynaud's phenomena. Capillaroscopic findings are accepted to be normal in primary Raynaud's phenomena, while capillaroscopic abnormalities are seen in scleroderma spectrum disorders. Several abnormalities may be also observed in other connective tissue diseases, such as systemic lupus erythematosus and antiphospholipid antibody syndrome. ${ }^{34,35}$ Capillaroscopic abnormalities (i.e., dilated capillaries) can be also seen in psoriatic skin. ${ }^{36}$ In the present study, none of the participants had inflammatory rheumatic 
conditions and dermatological diseases which would interfere with the results.

Nonetheless, there are some limitations to this study. First, the number of males is too small in our study which precludes the generalization of the results to the entire fibromyalgia population. Second, although the present study provides us data on microvascular changes in fibromyalgia, the cross-sectional design does not allow us to clarify whether microvasculopathy is a consequence or a cause of fibromyalgia.

In conclusion, patients with fibromyalgia show nailfold capillaroscopic changes including increased capillary loop diameter, increased number of micro-aneurysms, avascular areas, and neoangiogenic capillaries. However, capillaroscopic findings, in general, are not related to clinical variables and disease-related measures. The potential role of microvasculopathy in disease pathogenesis and treatment should be further studied.

\section{Declaration of conflicting interests}

The authors declared no conflicts of interest with respect to the authorship and/or publication of this article.

\section{Funding}

The authors received no financial support for the research and/or authorship of this article.

\section{REFERENCES}

1. Branco JC, Bannwarth B, Failde I, Abello Carbonell J, Blotman F, Spaeth M, et al. Prevalence of fibromyalgia: a survey in five European countries. Semin Arthritis Rheum 2010;39:448-53.

2. Lage-Hansen PR, Chrysidis S, Lage-Hansen M, Hougaard A, Ejstrup L, Amris K. Concomitant fibromyalgia in rheumatoid arthritis is associated with the more frequent use of biological therapy: a cross-sectional study. Scand $J$ Rheumatol 2016;45:45-8.

3. Macfarlane GJ, Pathan E, Siebert S, Packham J, Gaffney K, Choy E, et al. AxSpA patients who also meet criteria for fibromyalgia: identifying distinct patient clusters using data from a UK national register (BSRBR-AS). BMC Rheumatol 2019;3:19.

4. Coskun Benlidayi I. Fibromyalgia interferes with disease activity and biological therapy response in inflammatory rheumatic diseases. Rheumatol Int 2020;40:849-58.
5. Wolfe F, Clauw DJ, Fitzcharles MA, Goldenberg DL, Häuser W, Katz RL, et al. 2016 Revisions to the 2010/2011 fibromyalgia diagnostic criteria. Semin Arthritis Rheum 2016;46:319-29.

6. Morf S, Amann-Vesti B, Forster A, Franzeck UK, Koppensteiner R, Uebelhart D, et al. Microcirculation abnormalities in patients with fibromyalgia - measured by capillary microscopy and laser fluxmetry. Arthritis Res Ther 2005;7:R209-16.

7. Katz DL, Greene L, Ali A, Faridi Z. The pain of fibromyalgia syndrome is due to muscle hypoperfusion induced by regional vasomotor dysregulation. Med Hypotheses 2007;69:517-25.

8. Choi DH, Kim HS. Quantitative analysis of nailfold capillary morphology in patients with fibromyalgia. Korean J Intern Med 2015;30:531-7.

9. Lambova SN, Muller-Ladner U. Capillaroscopic findings in primary fibromyalgia. Curr Rheumatol Rev 2018;14:36-41.

10. Scolnik M, Vasta B, Hart DJ, Shipley JA, McHugh NJ, Pauling JD. Symptoms of Raynaud's phenomenon (RP) in fibromyalgia syndrome are similar to those reported in primary $\mathrm{RP}$ despite differences in objective assessment of digital microvascular function and morphology. Rheumatol Int 2016;36:1371-7.

11. Burckhardt CS, Clark SR, Bennett RM. The fibromyalgia impact questionnaire: development and validation. J Rheumatol 1991;18:728-33.

12. Sarmer S, Ergin S, Yavuzer G. The validity and reliability of the Turkish version of the Fibromyalgia Impact Questionnaire. Rheumatol Int 2000;20:9-12.

13. Beighton P, Solomon L, Soskolne CL. Articular mobility in an African population. Ann Rheum Dis 1973;32:413-8.

14. Grahame R, Bird HA, Child A. The revised (Brighton 1998) criteria for the diagnosis of benign joint hypermobility syndrome (BJHS). J Rheumatol 2000;27:1777-9.

15. Hosking SP, Bhatia R, Crock PA, Wright I, Squance ML, Reeves G. Non-invasive detection of microvascular changes in a paediatric and adolescent population with type 1 diabetes: a pilot cross-sectional study. BMC Endocr Disord 2013;13:41.

16. Sulli A, Secchi ME, Pizzorni C, Cutolo M. Scoring the nailfold microvascular changes during the capillaroscopic analysis in systemic sclerosis patients. Ann Rheum Dis 2008;67:885-7.

17. Etehad Tavakol M, Fatemi A, Karbalaie A, Emrani Z, Erlandsson BE. Nailfold Capillaroscopy in Rheumatic Diseases: Which Parameters Should Be Evaluated? Biomed Res Int 2015;2015:974530.

18. Bordy R, Totoson P, Prati C, Marie C, Wendling D, Demougeot C. Microvascular endothelial dysfunction in rheumatoid arthritis. Nat Rev Rheumatol 2018;14:404-20.

19. Saygin D, Highland KB, Tonelli AR. Microvascular involvement in systemic sclerosis and systemic lupus erythematosus. Microcirculation 2019;26:e12440. 
20. Tsilioni I, Russell IJ, Stewart JM, Gleason RM, Theoharides TC. Neuropeptides CRH, SP, HK-1, and Inflammatory Cytokines IL-6 and TNF Are Increased in Serum of Patients with Fibromyalgia Syndrome, Implicating Mast Cells. J Pharmacol Exp Ther 2016;356:664-72.

21. Mendieta D, De la Cruz-Aguilera DL, BarreraVillalpando MI, Becerril-Villanueva E, Arreola R, Hernández-Ferreira E, et al. IL-8 and IL-6 primarily mediate the inflammatory response in fibromyalgia patients. J Neuroimmunol 2016;290:22-5.

22. Kadetoff D, Lampa J, Westman M, Andersson $\mathrm{M}$, Kosek E. Evidence of central inflammation in fibromyalgia-increased cerebrospinal fluid interleukin-8 levels. J Neuroimmunol 2012;242:33-8.

23. Pernambuco AP, Schetino LP, Alvim CC, Murad CM, Viana RS, Carvalho LS, et al. Increased levels of IL-17A in patients with fibromyalgia. Clin Exp Rheumatol 2013;31(6 Suppl 79):S60-3.

24. Coskun Benlidayi I. Role of inflammation in the pathogenesis and treatment of fibromyalgia. Rheumatol Int 2019;39:781-91.

25. Imamura M, Targino RA, Hsing WT, Imamura S, Azevedo RS, Boas LS, et al. Concentration of cytokines in patients with osteoarthritis of the knee and fibromyalgia. Clin Interv Aging 2014;9:939-44.

26. Bote ME, García JJ, Hinchado MD, Ortega E. Inflammatory/stress feedback dysregulation in women with fibromyalgia. Neuroimmunomodulation 2012;19:343-51.

27. Malhotra D, Saxena AK, Dar SA, Kumar V, Nasare $\mathrm{N}$, Tripathi AK, et al. Evaluation of cytokine levels in fibromyalgia syndrome patients and its relationship to the severity of chronic pain. Journal of Musculoskeletal Pain 2012;20:164-9.

28. Xiao Y, He W, Russell IJ. Genetic polymorphisms of the beta2-adrenergic receptor relate to guanosine protein- coupled stimulator receptor dysfunction in fibromyalgia syndrome. J Rheumatol 2011;38:1095-103.

29. Iannuccelli C, Guzzo MP, Atzeni F, Mannocci F, Alessandri C, Gerardi MC, et al. Pain modulation in patients with fibromyalgia undergoing acupuncture treatment is associated with fluctuations in serum neuropeptide Y levels. Clin Exp Rheumatol 2017;35 Suppl 105:81-5.

30. Alcocer-Gómez E, Garrido-Maraver J, Bullón P, MarínAguilar F, Cotán D, Carrión AM, et al. Metformin and caloric restriction induce an AMPK-dependent restoration of mitochondrial dysfunction in fibroblasts from Fibromyalgia patients. Biochim Biophys Acta 2015;1852:1257-67.

31 Cordero MD, Díaz-Parrado E, Carrión AM, Alfonsi S, Sánchez-Alcazar JA, Bullón P, et al. Is inflammation a mitochondrial dysfunction-dependent event in fibromyalgia? Antioxid Redox Signal 2013;18:800-7.

32. Görlach A, Dimova EY, Petry A, Martínez-Ruiz A, Hernansanz-Agustín P, Rolo AP, et al. Reactive oxygen species, nutrition, hypoxia and diseases: Problems solved? Redox Biol 2015;6:372-85.

33. McGarry T, Biniecka M, Veale DJ, Fearon U. Hypoxia, oxidative stress and inflammation. Free Radic Biol Med 2018;125:15-24.

34. Cortes S, Cutolo M. Capillarosecopic patterns in rheumatic diseases. Acta Reumatol Port 2007;32:29-36.

35. Smith V, Herrick AL, Ingegnoli F, Damjanov $N$, De Angelis R, Denton CP, et al. Standardisation of nailfold capillaroscopy for the assessment of patients with Raynaud's phenomenon and systemic sclerosis. Autoimmun Rev 2020;19:102458.

36. De Angelis R, Bugatti L, Del Medico P, Nicolini M, Filosa G. Videocapillaroscopic findings in the microcirculation of the psoriatic plaque. Dermatology 2002;204:236-9. 